\title{
A structured multiblock compressible flow solver SPARTA for planetary entry probes
}

\author{
P. Papadopoulos \& P. Subrahmanyam \\ Department of Mechanical and Aerospace Engineering, \\ Center of Excellence for Space Transportation \& Exploration, \\ San Jose State University, San Jose, CA, USA
}

\begin{abstract}
SPARTA is a platform independent Graphical User Interface based two-dimensional compressible flow solver on multiple block structured grids that is developed and integrated to a planetary probe database to study trajectory, aerodynamic heating and flow-field analysis. It is a time-integration solver of the Navier-Stokes equations. The flow geometry may either be planar or axisymmetric. A comprehensive database of atmospheric entry vehicles and aeroshell configurations is developed. The database comprises vehicle dimensions, trajectory data, and aero-thermal, Thermal Protection Systems data for many different ballistic entry vehicles. Material properties for Carbon and Silicon based ablators are modeled and can be accessed from the database. SPARTA provides capabilities to choose from a list of flight vehicles or enter geometry information of a vehicle in design. A fourth order Runge-Kutta integration is employed for trajectory calculations. Fay-Riddell and Sutton-Grave empirical correlations have been used for the stagnation point convective heat transfer and Tauber-Sutton for the stagnation point Radiative heat transfer calculations. An approach is presented for dynamic TPS sizing. The inputs for the flow solver come from the trajectory output. SPARTA is a trajectory based flow solver.
\end{abstract}

Keywords: aerothermodynamics, Riemann solver, relational database, trajectory, compressible flow solver, planetary probes, convective and radiative aerodynamic heating, thermal protection systems, Navier-Stokes equations. 


\section{Introduction}

The Unites States national vision for space exploration calls for "The human and robotic exploration of the Solar System and beyond". Human and Robotic exploration of the solar system is to search for evidence of life, to understand the history of the solar system and to support human exploration. But before they arrive, planetary probes will likely have preceded them to provide the understanding required to make further exploration possible. Advances in technology ranging from new instrumentation, sophisticated materials and improvised nanotechnology makes planetary probes a vital tool in pursuit of scientific truth and the origins.

In support of this mission, a complete computational design framework is necessary that automates the calculations for stagnation point heating, TPS sizing and CFD calculations so that better probes can be built with this analysis. An integrated planetary probe design framework that automatically computes TPS sizing and convective stagnation point heating using Fay and Riddell [1] and radiative heating based on the Tauber-Sutton correlations [2] given vehicle geometry and entry trajectory flight conditions is presented in this publication. The probe design framework includes access to existing probe designs and provides a mini-CAD like design environment for construction of new configurations based on classes consistent with existing designs. A platform independent Graphical User Interface (GUI) based, relational database and trajectory driven CFD tool called SPARTA has been developed to accurately predict aerodynamic and heating entry environments. The aero-heating environment depends on the trajectory flown, size and shape of the vehicle. Trajectory driven CFD capability is demonstrated in this article.

\section{Approximate Riemann solver}

\subsection{Flow solver}

SPARTA is a compressible multi-block flow solver in two dimensions geometries: planar or axisymmetric. It is based on the cell-centered finite-volume formulation of the Navier-Stokes equations and has a shock capturing capability through the application of a limited reconstruction scheme and an upwind-biased flux calculator. SPARTA is capable of modeling flows that include shear layers, expansions, shocks and boundary layers. The governing equations are expressed in integral form over arbitrary quadrilateral cells with the time rate of change of conserved quantities in each cell specified a summation of the fluxes: mass, momentum and energy. The integral form of the Navier-Stokes equations in Cartesian coordinates is used as the starting point of the code formulation in the flow solver. The flow domain is discretized by a structured grid and a finitevolume approach is used to discretize the conservation equations. The flow field is recorded as cell-average values at cell centers and explicit time stepping is used to update conserved quantities. 
A Riemann solver is developed to calculate Inviscid fluxes across cell faces while central differences schemes are used to calculate the viscous fluxes. The governing equations for SPARTA is the set of Navier-Stokes equation which in integral form, can be expressed as

$$
\frac{\partial}{\partial t} \int_{V} U d V=-\int_{S}\left(\bar{F}_{i}-\bar{F}_{v}\right) \cdot \hat{n} d A+\int_{V} Q d V
$$

where $V$ is the cell's volume, $S$ is the bounding surface and $\hat{n}$ is the outward facing unit normal of the control surface. For two dimensional flows, $V$ is the volume per unit depth in the $z$-direction and $\mathrm{A}$ is the area of the cell boundary per unit depth $z$. The array of conserved quantities per unit volume is

$$
U=\left[\begin{array}{l}
\rho \\
\rho u_{x} \\
\rho u_{y} \\
\rho E \\
\rho f_{i s}
\end{array}\right]
$$

These elements represent mass density, $x$-momentum per volume, $y$-momentum per volume, total energy per volume and mass density of species is. The flux vector is divided into inviscid and viscous components and the inviscid components, in two dimensions is:

$$
\bar{F}_{i}=\left[\begin{array}{l}
\rho u_{x} \\
\rho u_{x}^{2}+p \\
\rho u_{y} u_{x} \\
\rho E u_{x}+p u_{x} \\
\rho f_{i s} u_{x}
\end{array}\right] \hat{i}+\left[\begin{array}{l}
\rho u_{y} \\
\rho u_{x} u_{y} \\
\rho u_{y}^{2}+p \\
\rho E u_{y}+p u_{y} \\
\rho f_{i s} u_{y}
\end{array}\right] \hat{j}
$$

The viscous component is

$$
\bar{F}_{v}=\left[\begin{array}{l}
0 \\
\tau_{x x} \\
\tau_{y x} \\
\tau_{x x} u_{x}+\tau_{y x} u_{y}+q_{x} \\
\rho f_{i s} \mu_{x, i s}
\end{array}\right] \hat{i}+\left[\begin{array}{l}
0 \\
\tau_{x y} \\
\tau_{y y} \\
\tau_{x y} u_{x}+\tau_{y y} u_{y}+q_{y} \\
\rho f_{i s} \mu_{y, i s}
\end{array}\right] \hat{j}
$$




\subsection{Gas models}

A number of gas models have been developed and included in SPARTA flow solver. These include perfect gas mixtures, perfect gases and also models for air and nitrogen in chemical equilibrium. These gas models can be chosen from the drop down menu in the graphical user interface and can be assigned to the flow solver for the vehicle in design.

\section{SPARTA software architecture}

Figure 1 shows the software architecture of SPARTA. Once the TPS Sizing is done, it can be automatically linked to CFD tools for grid generation and analysis. A comprehensive database of ballistic reentry vehicles has been developed using the Planetary Mission Entry Vehicles manual [3]. This includes vehicle dimensions and trajectory data for all the capsules that has been flown in the past. The SPARTA GUI provides the capability to choose from a list of flight vehicle geometric information and entry trajectories. This tool is intended as a preliminary design framework for planetary entry vehicle design. The overall software architecture is shown in Figure 1. The front GUI allows the user to provide inputs for trajectory and geometry. It is connected to an extensive planetary probe database and a Matlab computational engine, which is used to generate flight trajectory data. From the trajectory data, stagnation point aerodynamic heating and TPS size requirements are computed. TPS Sizing requires the selection of materials that effectively protect the vehicle during reentry. The database has several different categories of Ablators to choose from including Carbon, Reusable Composites, Carbon-Phenolic and Silicon based ablators. With the emerging Information Technology growth, heterogeneous systems are rapidly developing and this presents a need for distributed analysis system. So, with this in mind a platform independent tool has been developed. This architecture neutral code has the capability to execute in any platform for analysis without having to recompile.

\subsection{Graphical user interface}

The GUI based analysis tool was developed in the Matlab platform-independent environment. Figure 2, shows the probe design options provided in the SPARTA interface. A planetary probe can be chosen from the user interface Flight vehicle drop down menu. When the flight vehicle is chosen, appropriate initial trajectory and vehicle dimensions data are populated in the input boxes from the relational database. Values in the input boxes can be changed by the user or the populated values can be used to run the trajectory simulation. The user is also able to choose a stagnation point correlation, either Fay and Riddell [1] or Sutton and Graves [4] correlations from the interface. The user can-either choose a flight vehicle for trajectory analysis from the list of available probe designs in the planetary probe database as explained above or construct a new configuration. This is achieved by calling the Geometry Engine from within the GUI. After the 
vehicle is constructed, the trajectory is computed by calling the computational engine from within the GUI.

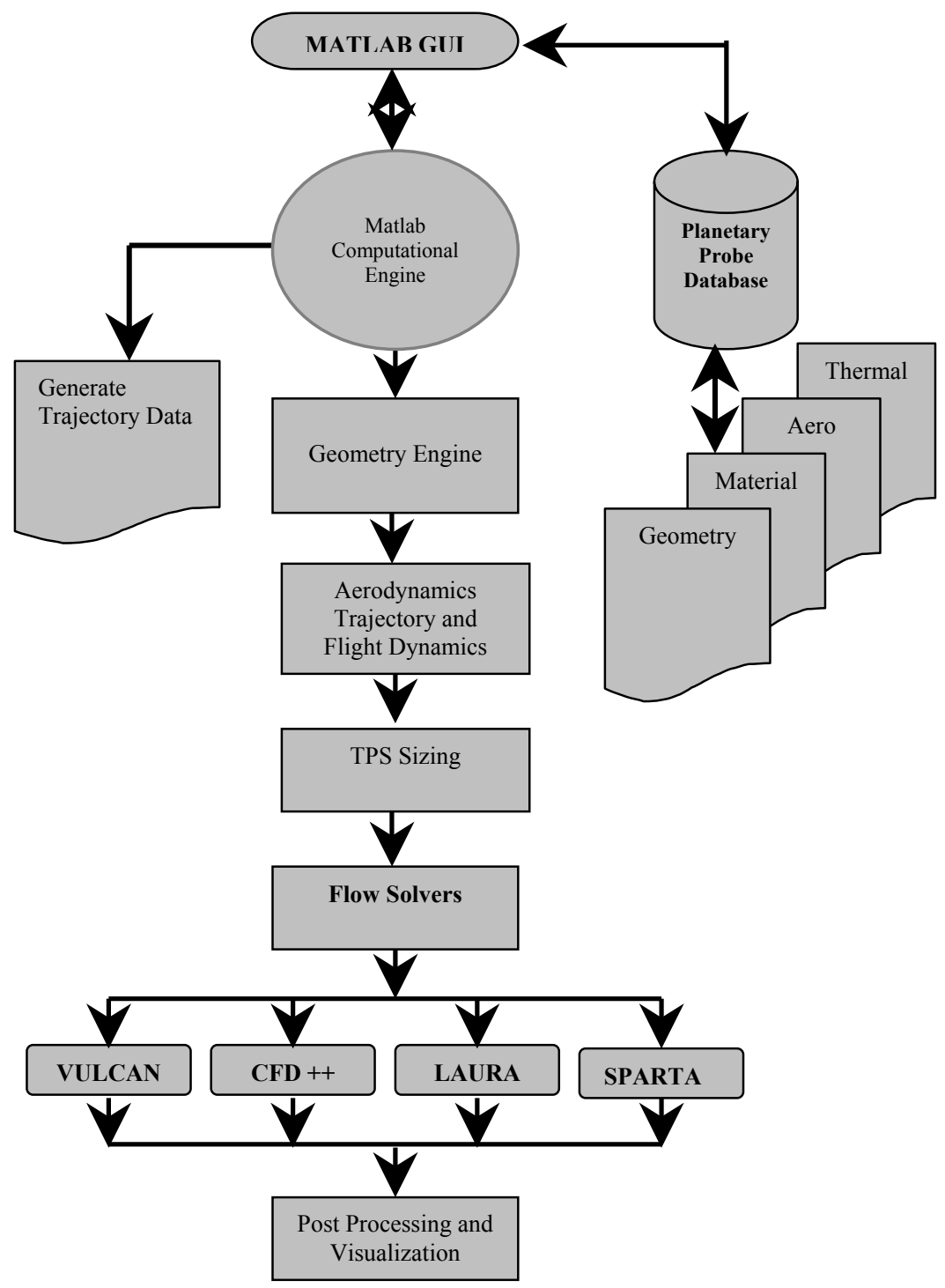

Figure 1: $\quad$ SPARTA software architecture.

\subsection{Atmospheric model}

SPARTA design environment also links the trajectory code to appropriate planetary empirical atmospheric models depending on the planetary probe that is 
chosen. The 1976 US Standard Atmosphere for Earth called GAME - General Atmospheric Model for Earth is modeled as a subroutine that calculates pressure, density, temperature, Reynolds number and speed of sound as a function of altitude. Global Reference Atmospheric Models (GRAM) [5] of Mars, Venus, Titan, and Neptune has been used to compute the atmospheric pressure, density and temperature profiles.

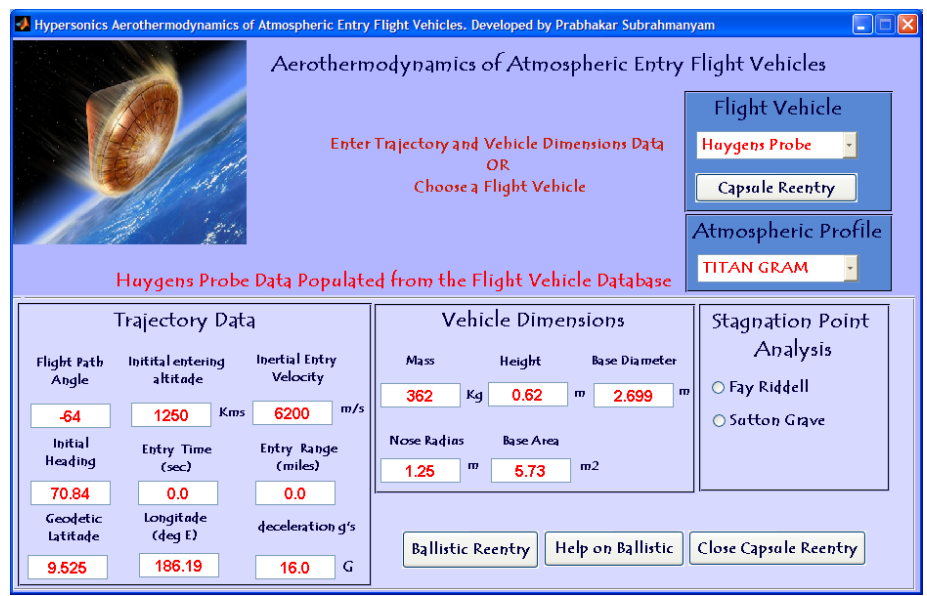

Figure 2: $\quad$ SPARTA graphical user interface.

\section{Trajectory driven CFD}

\subsection{Probe model}

The probe model developed for this trajectory is a point-mass model with two translations and one rotation (3-DOF) around a spherical planet. It integrates the equations of motions of a vehicle on a ballistic entry trajectory so that no lift is generated and the body acts only on gravity. The vehicle model is build from a number of parameters defining the geometry of the probe including body diameter, cone half-angle, nose and shoulder radius. The aerodynamic properties of the probe are subsequently derived from the geometry of the vehicle.

\subsection{Trajectory calculations}

The Apollo capsules were chosen for demonstration of the trajectory analysis capability. A sample trajectory data is shown in Table 1 and in figures 3(a) to $3(d)$.

\subsection{Test cases and CFD results}

The aeroshell analyzed is the Pathfinder configuration with a spherically blunted, $70^{\circ}$ cone with a nose radius $R_{N}$ of $0.020 \mathrm{~m}$, and the edge radius of the shoulder 
$\mathrm{R}_{\mathrm{S}}$ of $0.0020 \mathrm{~m}$. The freestream conditions are $\mathrm{P}_{\infty}=(7.1208)\left(10^{-5}\right) \mathrm{kg} / \mathrm{m}^{3}$, $\mathrm{T}_{\infty}=217 \mathrm{~K}, \mathrm{~V}_{\infty}=6571.7 \mathrm{~m} / \mathrm{s}$.

Table 1: $\quad$ Sample output of trajectory calculated by SPARTA.

\begin{tabular}{|c|c|c|c|c|c|c|}
\hline \multicolumn{7}{|c|}{ Trajectory Analysis for Flight Vehicle: Apollo 4} \\
\hline \multirow{2}{*}{$\begin{array}{l}\text { Altitude } \\
\text { (ft) }\end{array}$} & \multirow{2}{*}{$\begin{array}{l}\text { Velocity } \\
\text { (ft/sec) }\end{array}$} & \multirow{2}{*}{$\begin{array}{l}\text { Flight } \\
\text { Path } \\
\text { Angle } \\
\text { (deg) }\end{array}$} & & \multirow{2}{*}{$\begin{array}{l}\text { Mach } \\
\text { Number }\end{array}$} & \multirow{2}{*}{$\begin{array}{l}\text { Stag Point } \\
\text { Pressure } \\
\left(\mathrm{lbf} / \mathrm{ft}^{2}\right)\end{array}$} & \multirow{2}{*}{$\begin{array}{l}\text { Stag Point Heat } \\
\text { Transfer rate } \\
\dot{\boldsymbol{q}}_{\text {conv }}\left(\mathrm{W} / \mathrm{cm}^{2}\right)\end{array}$} \\
\hline & & & $\gamma$ & & & \\
\hline 250000.00 & 22500.00 & 12.00 & & 23.83 & 30.70 & 191.30 \\
\hline 249000.00 & 22501.37 & 12.00 & & 23.80 & 32.16 & 195.82 \\
\hline 248000.00 & 22502.75 & 12.01 & & 23.77 & 33.69 & 200.44 \\
\hline
\end{tabular}
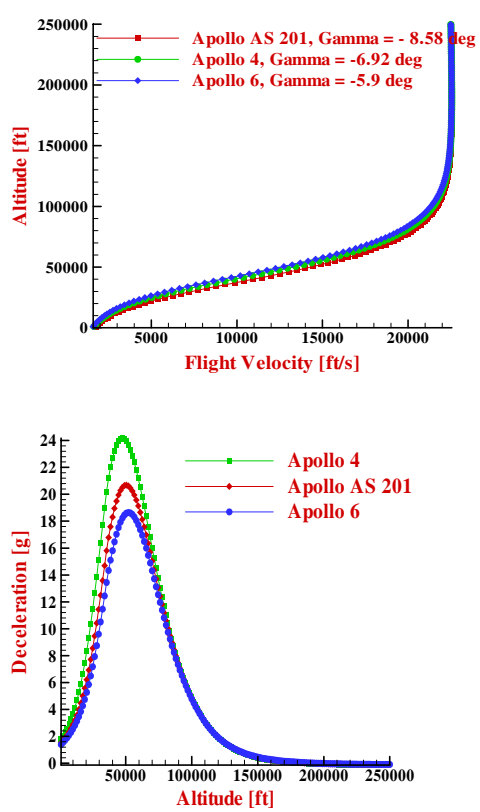
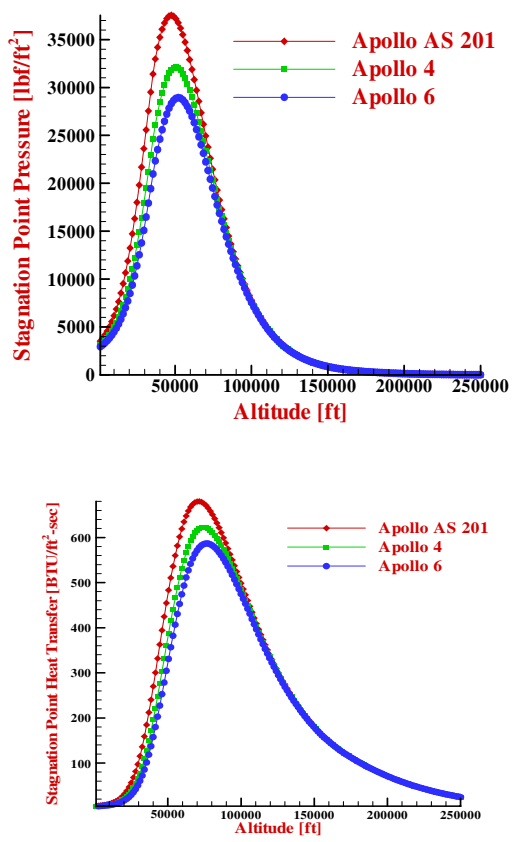

Figure 3: Trajectory data: flight velocity, Stagnation point heating, stagnation point pressure, deceleration vs. altitude.

\subsection{Planetary probe relational database and flight vehicle architecture}

The geometry of the flight vehicle is either constructed from the GUI for user specifications or generated from the SPARTA database for existing probes. The user specified inputs include vehicle dimensions such as the planetary probe's nose radius, forebody conical angle, corner radius, afterbody frustrum conical angle, and any additional base geometry specifications. Other design variables 
such as the probe mass, reference area and ballistic coefficient are also required to define the vehicle architecture. The user must also indicate the vehicle's angle-of-attack and freestream flight conditions along the reentry trajectory. A comprehensive database of existing planetary probe designs is provided in the SPARTA framework. Trajectory and geometry data are stored for each probe in the database. In addition to the capsule shapes, base areas, nose radii, payload masses and the ballistic coefficients of the probes are stored in the database. Pathfinder, Viking and Apollo class vehicle configurations can be generated from the database. The user can modify the existing vehicle designs by changing geometric features available in the GUI.
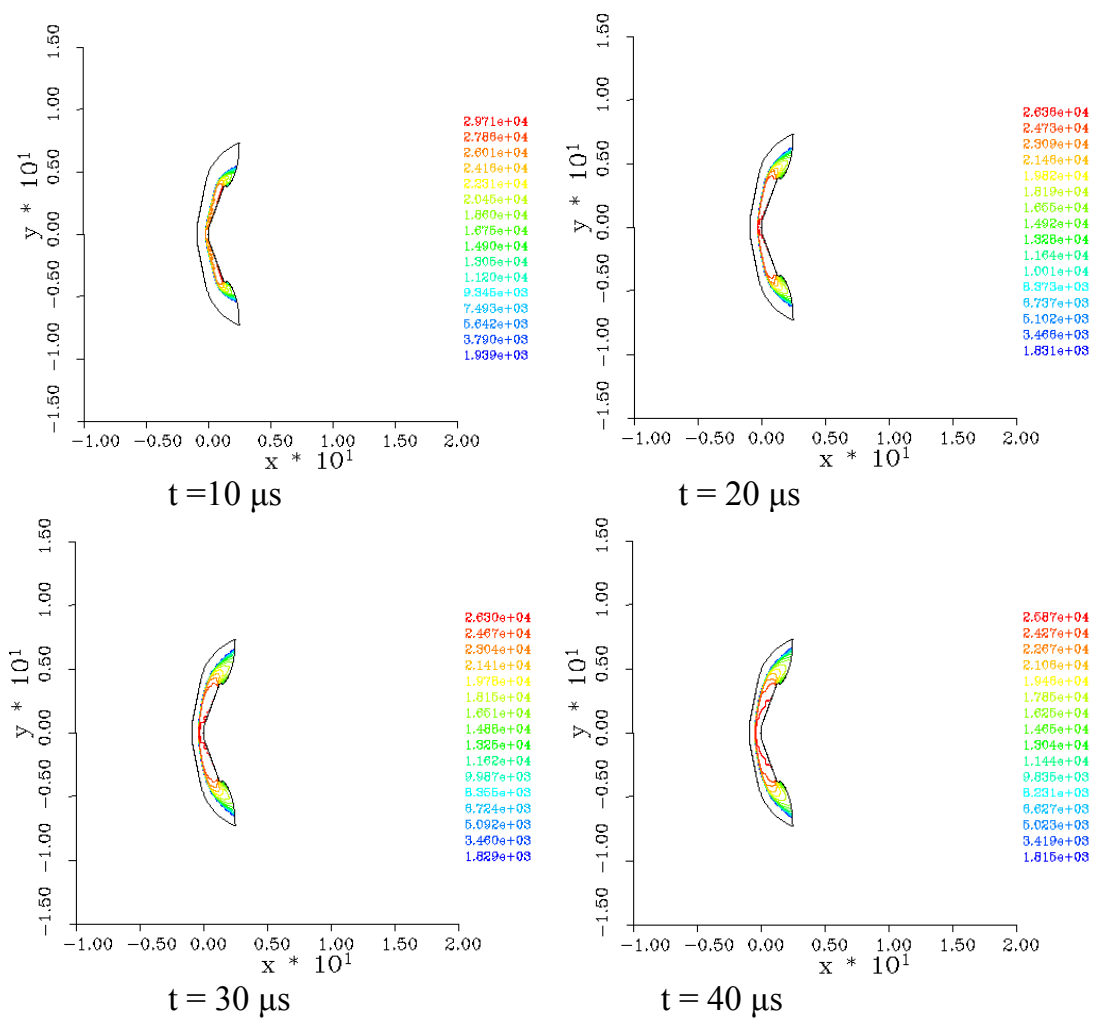

Figure 4: $\quad$ Transient flow over MARS Aeroshell at times $\mathrm{t}=10 \mu \mathrm{s}, 20 \mu \mathrm{s}, 30 \mu \mathrm{s}$ and $40 \mu \mathrm{s}$.

\section{Conclusions}

A trajectory based two-dimensional compressible flow solver CFD tool SPARTA is developed to solve multiple block structured grids. It is integrated to a comprehensive planetary probe database to study aerodynamic heating and flow-field analysis. It is a time-integration solver of the Navier-Stokes equations. 
The code calculates all flight conditions along the trajectories including aerodynamic and Aerothermodynamic stagnation point quantities. The code has been extensively benchmarked successfully against the industry standard trajectory codes. SPARTA is a platform-independent standalone Matlab based flight vehicle-database driven graphical user interface application to do the preliminary investigation of reentry vehicles. Appropriate atmospheric profiles have been developed and integrated with the code. For Venus, Mars and Neptune, the GRAM models have been used. Empirical correlations for estimating the stagnation point convective and radiative heat transfer have been modeled using Fay-Riddell and Tauber-Sutton correlations.

\section{References}

[1] J. Fay and F. Riddell, "Theory of Stagnation Point Heat Transfer in Dissociated Air" Journal of Aeronautical Sciences 25 (2), Feb 1958.

[2] Tauber, M. E., Sutton, K., "Stagnation Point Radiative Heating Relations for Earth and Mars Entries”, Journal of Spacecraft and Rockets, Vol 28, No 1, 1991, pp.40-42.

[3] C. Davies, "Planetary Mission Entry Vehicles," Quick Reference Guide, Ver. 2.1, NASA-ARC, 2002.

[4] K. Sutton, R.A Graves, "A General Stagnation Point Convective Heating Equation for Arbitrary Gas Mixture", NASA TR-376, 1971.

[5] Justus, C.G., and Johnson, D.L, Mars Global Reference Atmospheric Model 2001 Version (MARS- GRAM 2001) Users Guide, NASA/TM2001-210961, April 2001. 